\title{
STABILITY IN PARADIGM BIOLOGICAL SYSTEMS
}

\author{
ANDREW J. RIXON ${ }^{1}$, CRAIG R. JOHNSON ${ }^{1}$ and ALAN S. JONES ${ }^{1}$
}

(Received 19 February 1996; revised 20 December 1996)

\begin{abstract}
A discrete spatial model of a multi-species environment is formulated, and the behaviour of the system is studied. The model is used to explore stability and resilience of biological systems and discuss how they are dependent on spatial scale chosen.
\end{abstract}

\section{Introduction}

Nature is in constant change. Disturbances, such as a cyclone passing over a coral reef or fires in bushlands, lead to a change in the composition of an ecosystem. In turn, these changes impact upon the stability of the system.

Recent works $[5,14,20]$ have suggested the existence of multiple stable states within the dynamic of model ecosystems. Hence, the system will almost surely not revert to its original form. This poses the question of how properly to specify 'stability' of such a system, and what constitutes a 'recovery'.

The traditional approach to the concept of stability of a system is that of mathematical stability. Here one identifies the equilibrium points of the system and then by local analysis around these points, deduces whether or not the system is stable.

Ecologists and biologists find it difficult to deal usefully with this form of stability when analysing the dynamics of an ecosystem. As has been mentioned above, a system may have many equilibrium points to which it moves under perturbation. Mathematically, this system may be classified as being unstable. However its ability to 'recover' to an ecosystem with similar assemblages as before, would lead an ecologist to declare the system stable.

The notion of resilience [13] seems to be a much more useful concept for describing the dynamics of an ecosystem. Here resilience is a measure of the persistence of systems and of their ability to absorb change and disturbance and still maintain the same relationships within their population. It is, the basic property of a system

'Department of Mathematics, University of Queensland, St Lucia Qld 4072, Australia

(C) Australian Mathematical Society, 1998, Serial-fee code 0334-2700/98 
maintaining established relationships among its parameters.

An important factor which has been noted with regards to a system's stability is spatiality $[2,8]$. Here the effects of spatial variability can stabilize an otherwise unstable model ecosystem.

What follows within this paper is a formulation of a discrete spatial model which will be shown to have an equilibrium space. Stability of the model will be shown to be dependent on the spatial scale chosen. The stability of the system is judged by the ability of the system to maintain a non-trivial assemblage after perturbation. When the model is iterated, we say that the system has formed a trivial ássemblage if the collection of species collapses into a mono-culture.

Thus if the system is resilient to the perturbations introduced we can conclude that our system is in fact stable. We shall see that the resilience of the system is dependent on the spatial scale of the model.

\section{The model}

Cellular automata are a powerful class of simulation models for explicitly spatially functioning ecosystems, such as the competition between species on a coral reef. Such a reef consists of millions of polyps, so that attempting to model the complete system is computationally inefficient. Instead we have taken a smaller section, and identified the boundaries (forming a torus) in order to avoid edge effects. However, running the program with finite boundary conditions does not significantly alter the results for reasonably sized grids. Within this lattice structure we apply our specific neighbourhood form and transition function.

Symbolically we can represent the new state of the center cell in the neighbourhood $a_{c}^{t+1}$ being dependent on the specific neighbourhood chosen with the transition function $\Phi$ operating on the cells in this neighbourhood $a_{\epsilon}^{t}$. That is, $a_{c}^{t+1}=\Phi\left(a_{\epsilon}^{t}\right)$.
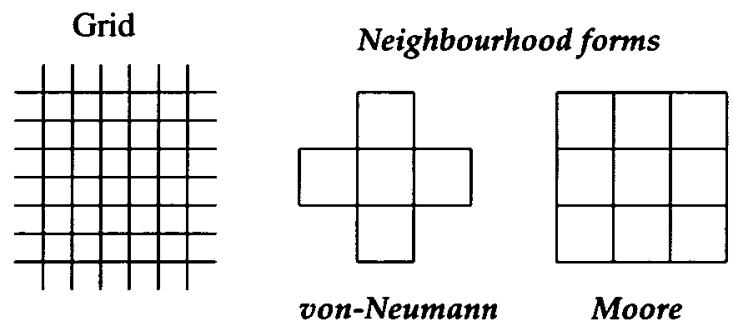

FIGURE 1. Common neighbourhood forms applied to a grid structure.

In the model being considered here, the transition function which operates on the cell states within the certain neighbourhood form chosen is a particular rule called an 
intransitive rule system. We have four species which are linked together in a certain chain of interaction. This defines which species will outcompete/overgrow the other species within its neighbourhood. This model then simulates a possible assemblage on a coral reef where certain species are better competitors than others.

The rule which is deterministically applied to this neighbourhood throughout the grid is described as below. Numbers one through to four represent the four species which can populate the environment in this model.
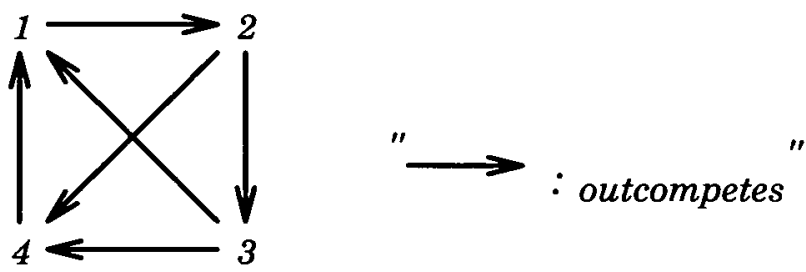

FIGURE 2. Intransitive Model.

Once the grid size is chosen, initialisation of the grid guarantees that there is a complete fill of all sites within the grid structure with one of the four possible species.

Below is a simple worked example where we chose a grid size of $3 \times 3$, and then apply our algorithm. To see how the updating works for each generation we take two cases.

Initial grid structure

$$
\begin{aligned}
& \text { Neighbourhood of } \\
& \text { row } 1 \text {, col } 1 \text { element }
\end{aligned}
$$

\begin{tabular}{|l|l|l|}
\hline 1 & 2 & 3 \\
\hline 3 & 4 & 1 \\
\hline 3 & 2 & 1 \\
\hline
\end{tabular}

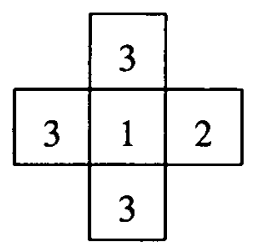

Here species 3 wins the site of species 1 .
Neighbourhood of row 2 , col 2 element

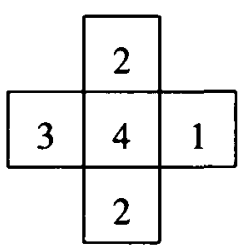

Here species 2 wins the site of species 4 .

FIGURE 3. Worked example.

First case we take the row 1, col 1 element and apply the Von-Neumann neighbourhood to see what its immediate neighbours are. From this we then infer which species number will survive according to the rule structure as described above. Here we can see that the only species which outcompetes species 1 is species 3 and hence it is 3 which becomes the new owner of this site. 
Another interesting example of the rule structure is when we consider element at row 2, col 2 where species 4 is in the neighbourhood structure as noted in diagram. From the rule structure we can see that there are two species which outcompete 4 , those being species 2 and 3 . However it can be seen that species 2 outcompetes species 3 and hence species 2 wins the site.

Thus after one iteration it can be verified that our initial starting configuration transforms into the new environment as pictured below.

\begin{tabular}{|l|l|l|}
\hline 1 & 2 & 3 \\
\hline 3 & 4 & 1 \\
\hline 3 & 2 & 1 \\
\hline
\end{tabular}

\begin{tabular}{|l|l|l|}
\hline 3 & 1 & 2 \\
\hline 3 & 2 & 3 \\
\hline 2 & 1 & 3 \\
\hline
\end{tabular}

FIGURE 4. Complete update of environment.

\section{Behaviour of the model}

Having formulated our model we can discuss the dynamical behaviour of the system under iteration. If the system collapses to a mono-culture then we define this to be a fixed point, otherwise the system will fall into a cyclical equilibrium. We call the set of all fundamental attractors to which the system will fall its 'equilibrium space'.

We shall see that the time taken to reach these equilibria is comparatively small but that as one increases the grid size, the number of iterations to equilibria increases slightly.

Finally, as one increases the grid size it will be apparent that there is an decreased chance of the system forming a mono-culture. This shows that for larger chosen grid sizes there is a greater chance of a non-trivial ecosystem assemblage forming. That is, the chance for species co-existence is high for large grid sizes.

3.1. Equilibrium space The underlying deterministic algorithm and finite state space in which the system evolves is responsible for the basic type of equilibria always formed. We define the collection of basic attractors for a specified grid size its equilibrium space. The number of attractors in this set is dependent on the grid size chosen.

To look in a little more depth at the notion of equilibrium space we can study simple examples such as a grid size of $2 \times 2$ or $3 \times 3$. One of the problems with trying to explore all possibilities of these spatial systems is that computationally the problem increases exponentially. An upper bound for the number of starting states (initial configurations) 
can be expressed by $4^{k^{2}}$ where $k$ is the row size of a square grid. Hence it becomes obvious that for larger grid sizes the only feasible technique of enquiry is that a Monte-Carlo type simulation.

For a $2 \times 2$ case the set of attractors, 'Equilibrium space', can be seen as pictured below. All possible initial starting configurations fall into one of the seven basic non-trivial attractors or into one of the four trivial fixed points. There is a high bias towards mono-cultures at this level indicating instability.

Non-trivial period 4 attractors.

\begin{tabular}{|l|l|}
\hline 1 & 2 \\
\hline 4 & 3 \\
\hline
\end{tabular}$\rightarrow$\begin{tabular}{|l|l|}
\hline 4 & 1 \\
\hline 3 & 2 \\
\hline
\end{tabular}$\rightarrow-$\begin{tabular}{|l|l|}
\hline 3 & 4 \\
\hline 2 & 1 \\
\hline
\end{tabular}$\rightarrow-$\begin{tabular}{|l|l|}
\hline 2 & 3 \\
\hline 1 & 4 \\
\hline
\end{tabular}

\begin{tabular}{l|l|}
\hline 3 & 1 \\
\hline 3 & 2 \\
\hline
\end{tabular}$\rightarrow-$\begin{tabular}{|l|l|}
\hline 3 & 3 \\
\hline 2 & 1 \\
\hline
\end{tabular}$\rightarrow-$\begin{tabular}{|l|l|}
\hline 2 & 3 \\
\hline 1 & 3 \\
\hline 2 & 2 \\
\hline 1 & 4 \\
\hline
\end{tabular}$\rightarrow-$\begin{tabular}{|l|l|l|}
\hline 1 & 2 \\
\hline 3 & 3 \\
\hline 4 & 2 \\
\hline
\end{tabular}$\rightarrow-$\begin{tabular}{|l|l|}
\hline 2 & 1 \\
\hline 2 & 2 \\
\hline 2 & 1 \\
\hline
\end{tabular}

Mono-cultures
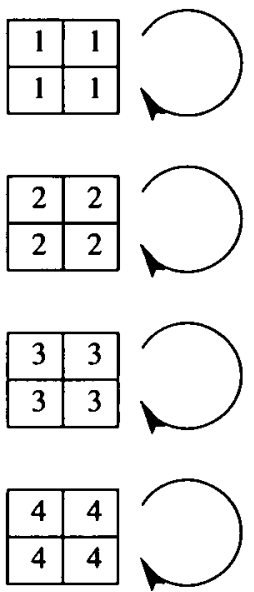

\begin{tabular}{|l|l|}
\hline 4 & 4 \\
\hline 1 & 2 \\
\hline
\end{tabular}$\rightarrow$\begin{tabular}{|l|l|}
\hline 4 & 2 \\
\hline 4 & 1 \\
\hline 2 & 2 \\
\hline 1 & 3 \\
\hline
\end{tabular}$\rightarrow-$\begin{tabular}{|l|l|l|}
\hline 2 & 1 \\
\hline 4 & 4 \\
\hline 3 & 2 \\
\hline
\end{tabular}$\rightarrow-$\begin{tabular}{|l|l|l|}
\hline 3 & 1 & 4 \\
\hline 2 & 4 \\
\hline 2 & 2 \\
\hline
\end{tabular}

FIGURE 5. Equilibrium space of $2 \times 2$ system.

It is interesting to note that all the cycles formed here for the $2 \times 2$ are of period 4 . It shall be seen that when looking at grid size of $3 \times 3$ and over there are a variety of different periods formed.

When we increase the grid size to $3 \times 3$ the number of attractors in the equilibrium space is now 1027, with 1023 non-trivial assemblages and 4 mono-cultures. The number of attractors continues to grow exponentially as one proceeds to increase the 
grid size. This increase of attractors within the system will be seen to be the reason for a decline in mono-cultures being formed as the grid size is increased.

3.2. Settling time The average number of iterations taken to reach equilibrium is an important gauge of the systems stability when we are considering its response to perturbation. If a system is able to recover rapidly after perturbation then obviously this has a higher degree of stability then a system which takes a long time to regain a functional assemblage.

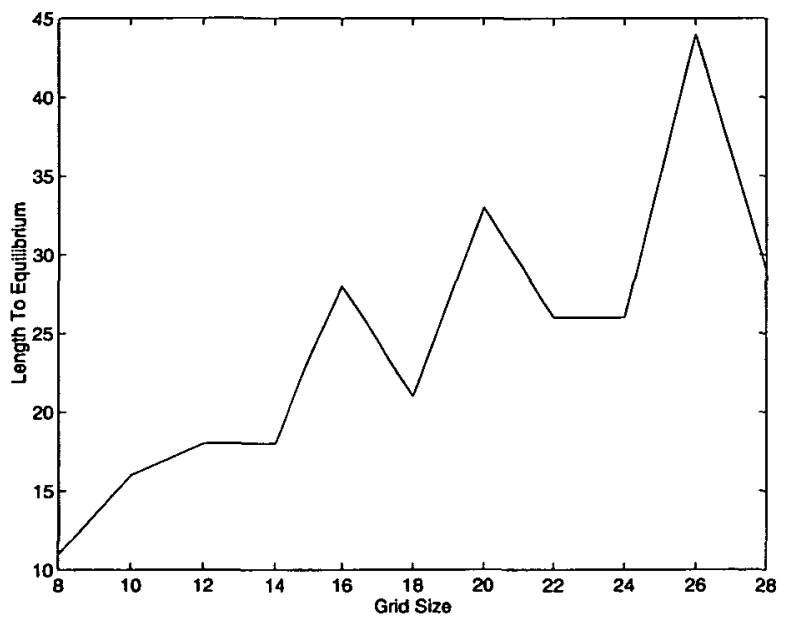

FIGURE 6. Stepwise increase in average length to equilibrium.

It can be seen from the graph that there is a kind of step function underlying the increase in the average number of iterations taken to reach equilibrium. ${ }^{2}$

The deviation in equilibrium times for different realizations for a $12 \times 12$ grid can be seen from the table below.

If one perturbs the system which is at equilibrium the time taken to reach a new equilibrium is much less then the system starting from a random configuration.

3.3. Decrease in mono-cultures A result which reflects upon systems ability to establish itself, is how the frequency of fixed points (mono-cultures) is affected by grid size. The table below shows that the relative number of starting configurations which collapse to a mono-culture declines quite rapidly as one increases grid size, thus indicating the greater chance of a non-trivial ecosystem assemblage for larger chosen grid sizes.

Since our system is spatial we have to generate new spatial configurations to iterate

${ }^{2}$ All results are obtained by starting the model in a random configuration and then letting it run until equilibrium is reached. 
TABLE 1. Variations in different realisations.

\begin{tabular}{|c|c|}
\hline Period Found & Length to equilibrium \\
\hline \hline 3 & 19 \\
6 & 15 \\
3 & 11 \\
3 & 21 \\
3 & 20 \\
3 & 14 \\
3 & 17 \\
6 & 19 \\
\hline
\end{tabular}

and from this we extract our data. Here the number of configurations processed represents the number of ways to assign species one through to four in a relationship so as to fill the entire grid size chosen. That is, $S_{1}+S_{2}+S_{3}+S_{4}=G^{2}$, where $S_{i}$ represents each species and $G^{2}$ represents the number of cells in a particular grid size. Once the number of each species has been chosen so as to satisfy this relation, the grid is then filled at random with each these species.

TABLE 2. Collapse to mono-cultures dependent on grid size.

\begin{tabular}{|c|c|c|}
\hline Grid Size & $\begin{array}{c}\text { Percentage Collapse } \\
\text { To Fixed Points }\end{array}$ & $\begin{array}{c}\text { Number of } \\
\text { Configurations processed }\end{array}$ \\
\hline \hline $2 \times 2$ & 85.74 & 35 \\
$3 \times 3$ & 54.54 & 220 \\
$4 \times 4$ & 38.28 & 969 \\
$5 \times 5$ & 30.06 & 3276 \\
$6 \times 6$ & 24.01 & 9139 \\
$7 \times 7$ & 18.62 & 22100 \\
$8 \times 8$ & 14.44 & 47905 \\
$9 \times 9$ & 12.67 & 95284 \\
\hline
\end{tabular}

The reason behind the shrinkage in mono-cultures as grid size is increased is due to the fact that the number of attractors in the equilibrium space is increasing exponentially and hence there is a greater chance of the system falling into a nontrivial species co-existence.

It should be noted that the periodicity of the model is unaffected by choice of toroidal or open boundary conditions. The number of attractors in the equilibrium space decreases with open boundaries, but the basic dynamic of the system remains the same. Similarly, with different choices of neighbourhood rules, the periodic nature 
still remains under this particular rule structure. So it is the rule structure of the model which governs the behaviour rather then the type of boundary conditions or neighbourhood rule.

\section{Effect of perturbation}

Having explored how our model settles into equilibrium states, we are now able to observe what happens when we start perturbing our model once it has reached an equilibrium state. Within our model we simulate a perturbation by randomly assigning different species to, about $10 \%$ of the lattice site, at equilibrium, and then allowing the model to run.

Below shows such an example for a $15 \times 15$ grid where perturbations occur at multiples of 50 generations.

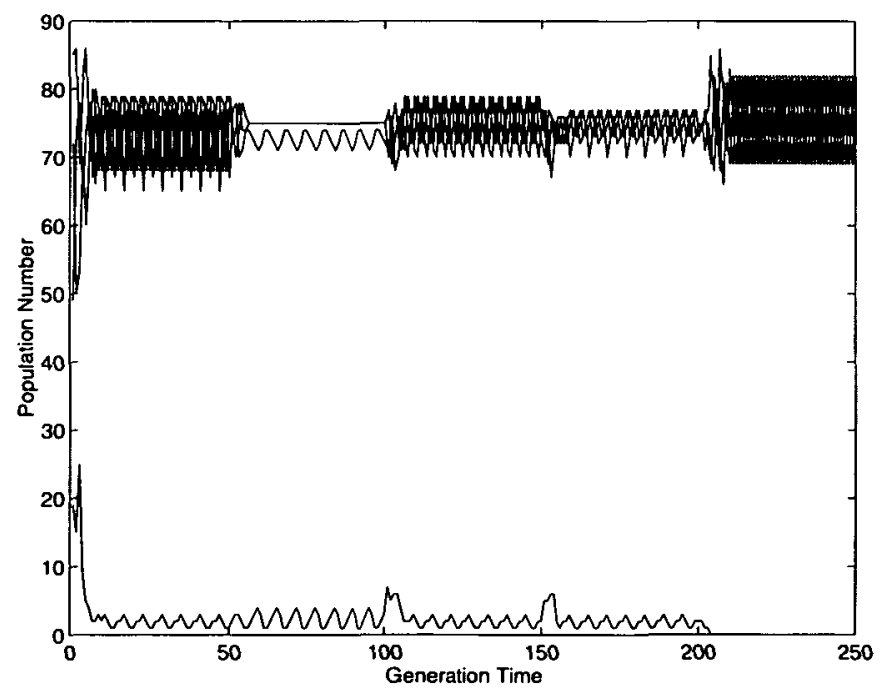

FIGURE 7. 15x15 system showing effect of Perturbation.

The cluster of jagged lines in the figure represent a clumping of 3 species forming a certain periodic cycle. The single line near the $x$-axis represents the population numbers of the fourth species, this too has formed a periodic equilibrium. It can be seen that the time taken for the system to recover after the perturbations is minimal indicating a high degree of stability.

After many simulations for a $15 \times 15$ grid the 3 species equilibria is found to be highly resilient to perturbation, with the 3 species always reforming a periodic cycle and hence maintaining co-existence. 
Occasionally, there can be 4 species co-existence. However this assemblage is highly unstable since it is easily destroyed by perturbation.

The question of how resilience is dependent on grid size, follows naturally after observing certain simulations for different grid sizes.

By using a Monte-Carlo simulation and allowing the system to settle into a equilibrium before perturbing the system, we obtain the following graph describing the percentage of simulations which collapse to a mono-culture as a function of the grid size.

As the grid size is increased the percentage of collapses to a mono-culture after perturbation decreases rapidly. This represents an increased resilience of the system with an increase in the grid size chosen.

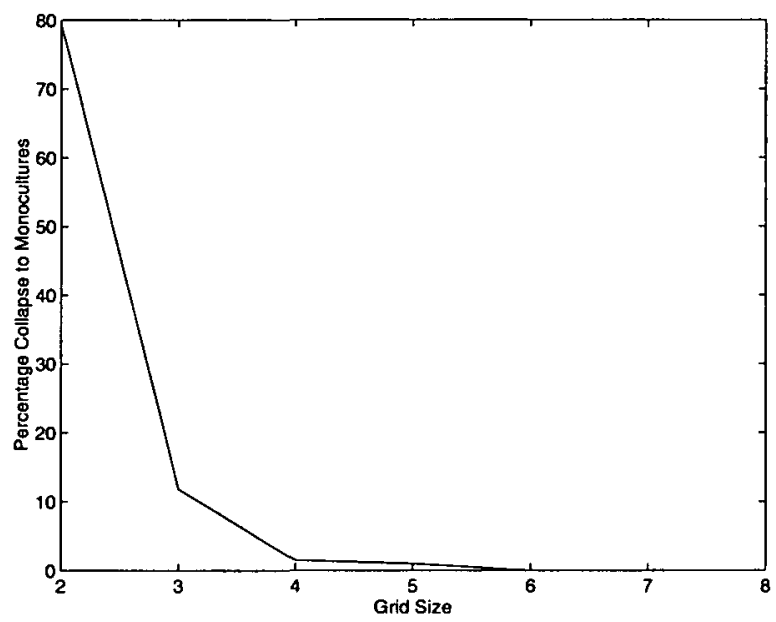

FIGURE 8. Percentage collapse to mono-culture after perturbation.

The system attains a highly resilient character once the grid size is chosen above $6 \times 6$. Once a system of this grid size or greater has reached an equilibria and is perturbed it will always fall back into a periodic equilibrium. This shows how the system has attained a buffering effect to disturbances and is able to maintain a non-trivial resilient ecosystem.

\section{Resilience}

In previous sections we have mentioned the notion of resilience and how the system exhibits the trait of being resilient to perturbation by maintaining the same assemblages before and after the perturbation. We have seen from Figure 7 how a $15 \times 15$ system responds to perturbation, and how it is able to maintain a non-trivial three species co- 
existence throughout perturbation. Four species co-existence occurs but is unstable since it is easily destroyed by perturbation. Also the collapse of an ecosystem after perturbation has been shown by Figure 8 to decrease as one increases the grid size. All reflecting on the systems ability to deal with disturbances.

It is useful to consider the simple case of a $2 \times 2$ grid size, to understand how this type of stability is obtained within the model. However it must be noted from Figure 8 that the $2 \times 2$ case is the most unstable ecosystem assemblage with $79 \%$ of simulations collapsing to a mono-culture after a perturbation.

For a certain grid size we can represent the spatial arrangement by a binary number which can then be converted back into decimal.

EXAMPLE 5.1. A $2 \times 2$ grid as pictured in Figure 9 can be represented by the binary number 00011011 which is 27 in decimal. It can be verified then that the complete iteration (represented in decimal) for this initial configuration is $27,82,100,133,25$, 82 thus forming a period four cycle taking one time step to reach this equilibrium.

Represent Species as:

\begin{tabular}{|l|l|}
\hline 1 & 2 \\
\hline 3 & 4 \\
\hline
\end{tabular}

$$
\begin{aligned}
& 1=00 \\
& 2=01 \\
& 3=10 \\
& 4=11
\end{aligned}
$$

FIGURE 9. Conversion to binary for a particular configuration.

The way that this relates to the notion of resilience can be seen from certain clumpings within the data for this $2 \times 2$ system.

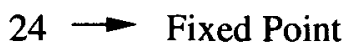

$$
\begin{aligned}
& 25,82,100,133,25 \\
& 26,98,164,137,26 \\
& 27,82,100,133,25,82 \\
& 28 \rightarrow \text { Fixed Point }
\end{aligned}
$$

FIGURE 10. Iteration data for a $2 \times 2$ system.

What this represents then is that if the bottom right corner cell as pictured below is either species 1,2 , or 3 then the outcome is the same, that being a periodic cycle.

That is, for this assemblage, provided the perturbation is minimal and in the right hand bottom corner, we can have the system maintaining a non-trivial ecosystem. 


\begin{tabular}{|l|l|}
\hline 1 & 2 \\
\hline 3 & $*$ \\
\hline
\end{tabular}

Where '*' can be either $4,3,2$

FIGURE 11. Iteration data for a $2 \times 2$ system.

For our $2 \times 2$ system a group of 3 elements clumped is the greatest that is to be found. When one increases the dimension to $3 \times 3$ grid size, there is a drastic increase in the clumping, with a group of up to 63 elements clumped.

Like for our $2 \times 2$ system, what this large clumping of elements means for a $3 \times 3$ system is an increase in the number of cells which can be occupied by a number of species and still result in a periodic equilibria being formed.

In terms of resilience to perturbation, what this means is that as the grid size of the system is increased there is a greater number of cells which are able to be occupied by a number of species and still maintain a non-trivial ecosystem. The mechanism of perturbation chosen for this model simulates a natural disturbance, such as a crown of thorns outbreak in a reef which 'eats' the corals resulting in different species growing back in the previous site.

Thus when our system attains an ability to have grid sites which are independent of the species which inhabits it, we have then formed a system which can be resilient to the type of perturbations introduced to our model.

\section{Conclusion}

To summarise, we have seen how the resilience of the system is dependent on the size of the grid chosen for our model. The model has shown a tendency for the four species co-existence to be unstable, with the fourth species being easily destroyed by perturbation. The resulting 3-species ecosystem formed has the property of being stable to perturbation provided that the spatial scale is large enough.

The model has been shown to have a set of fundamental attractors which increase exponentially as the grid size is increased. This is responsible for the system being able to form a non-trivial ecosystem as the grid size is increased. The basic dynamic of this model is unaffected by the choice of toroidal or open boundary configurations. Similarly the choice of neighbourhood interaction has no effect on the periodicity of the model. The results hold true over a variety of 'boundary conditions'.

Thus for our model the ability to be stable to perturbation, and exhibit a type of buffering effect to disturbances, yielding resilience, is dependent on the spatial scale being sufficiently large. 


\section{References}

[1] W. Brown, "Cheat thy neighbour - a recipe for success", New Scientist 9 (1993) 19-20.

[2] H. N. Commins, R. M. May and M. P. Hassel, "Spatial structure and chaos in insect population dynamics", Nature 353 (1991) 255-258.

[3] J. H. Connel and W. P. Sousa, "On the evidence needed to judge ecological stability or persistence", American Naturalist 121 (1983) 789-824.

[4] P. Davis, "Order in evolutionary chaos", Guardian Weekly 23 Jan (1994).

[5] H. T. Dublin, A. R. E. Sinclair and J. McGlade, "Elephants and fire as causes of multiple stable states in Serengeti-Mara woodlands", J. Animal Ecology 59 (1990) 1147-1164.

[6] M. J. Feigenbaum, "Universal behaviour in non-linear systems", Physica 7D (1983) 16-39.

[7] B. S. Goh, "Global stability in two species interactions", J. Math. Biol. 3 (1976) 313 - 318.

[8] A. Hastings, "Food web theory and stability", Ecology 69 (1988) 1665-1668.

[9] A. Hastings and K. Higgins, "Persistence of transients in spatially structured ecological models", Science 263 (1994) 1133-1136.

[10] R. H. G. Helleman, G. Iooss and R. Stora, "Chaotic behaviour of deterministic systems", Les Houches 85-134.

[11] P. Hogeweg, "Cellular automata as paradigm for ecological modelling", Appl. Math. Comp. 27 (1988) 81-100.

[12] A. V. Holden, Chaos (Manchester University Press Publications, 1987).

[13] C. S. Holling, "Resilience and stability of ecological systems", A. Rev. Ecol. Syst. 4 (1973) 1-23.

[14] N. Knowlton, "Thresholds and multiple stable states in coral reef dynamics", Amer. Zool. 32 (1990) 674-682.

[15] C. G. Langton, "Studying artificial life with cellular automata", Physica 22D (1986) 120-149.

[16] R. C. Lewontin, "The meaning of stability", Brookhaven Symp. Biol. 22 (1969) 13 - 23.

[17] R. M. May, "Stability and complexity in model ecosystems", Nature 238 (1972) 413-414.

[18] R. M. May, “Will a large complex system be stable?”, Nature 238 (1972) 413-414.

[19] R. M. May, "Simple mathematical models with very complicated dynamics", Nature 261 (1976) 459-466.

[20] R. M. May, "Thresholds and breakpoints in ecosystems with a multiplicity of stable states", Nature 269 (1977) $471-477$.

[21] R. M. May and M. A. Novak, "Evolutionary games and spatial chaos", Nature 359 (1992) 826-829.

[22] R. M. May and M. A. Novak, "Spatial dilemmas of evolution", Int. J. Bifurcation and Chaos in applied Sciences and Engineering 3 (1993) 35-78.

[23] C. H. Peterson, "Does a rigorous criterion for environmental identity preclude the existence of multiple stable points?", Am. Nat. 132 (1984) 652-661.

[24] S. L. Pimm, "Food web design and the effect of species deletion", OIKOS 35 (1980) 139-149.

[25] I. Stewart, Does God Play Dice? (Penguin Publications, 1989).

[26] J. P. Sutherland, "Perturbations, resistance, and alternative views of the existence of multiple stable points in nature", Am. Nat. 136 (1990) 270-275.

[27] S. Wolfram, "Cellular automata as models of complexity", Nature 311 (1984) 419-424. 